\title{
Frontières
}

\section{Un imaginaire de la fin cinématographique. Lecture croisée de The Tree of Life de Terence Malick et de Melancholia de Lars von Trier}

\section{Bertrand Gervais}

Volume 25, numéro 2, 2013

Apocalypses et imaginaires de la fin

URI : https://id.erudit.org/iderudit/1024942ar

DOI : https://doi.org/10.7202/1024942ar

Aller au sommaire du numéro

Éditeur(s)

Université du Québec à Montréal

ISSN

1916-0976 (numérique)

Découvrir la revue

Citer cet article

Gervais, B. (2013). Un imaginaire de la fin cinématographique. Lecture croisée

de The Tree of Life de Terence Malick et de Melancholia de Lars von Trier.

Frontières, 25(2), 109-120. https://doi.org/10.7202/1024942ar
Résumé de l'article

L'industrie cinématographique nous a donné, ces dernières décennies, une quantité impressionnante de films à caractère apocalyptique. Ces films couvrent l'ensemble des situations associées à une fin du monde, depuis les premières annonces de la fin jusqu'au monde d'après la fin. Les films oscillent entre des Apocalypses traditionnelles et des apocalypses intimes, de ces fins vécues sur un mode restreint et qui condensent le destin de l'humanité entière. Des films tels que The Tree of Life de Terence Malick (2010) et Melancholia de Lars von Trier (2011), qui seront décrits ici, sont de telles apocalypses intimes qui se tiennent en retrait des spectacles à grand déploiement. Malgré tout, d'importances différences les séparent, surtout en ce qui a trait à leur traitement de la fin et de ses significations. Car, si le premier s'ouvre sans compromis à un imaginaire chrétien, où la fin est envisagée dans la perspective d'une renaissance à Dieu, suggérant de façon à peine voilée que la vie se poursuit après la mort, le second engage une posture résolument athée et déclare plutôt, dans un matérialisme radical, que toute vie cesse une fois la mort venue. Ces films engagent des imaginaires de la fin distincts, l'un, un imaginaire traditionnel à bien des égards et teinté de transcendance, l'autre, un imaginaire contemporain, de facture nihiliste.
Ce document est protégé par la loi sur le droit d'auteur. L'utilisation des services d'Érudit (y compris la reproduction) est assujettie à sa politique d'utilisation que vous pouvez consulter en ligne.

https://apropos.erudit.org/fr/usagers/politique-dutilisation/ 


\section{UN IMAGINAIRE DE LA FIN CINÉMATOGRAPHIQUE. LECTURE CROISÉE DE THE TREE OF LIFE DE TERENCE MALICK ET DE MELANCHOLIA DE LARS VON TRIER}

Bertrand Gervais, Ph. D.

Professeur, Département d'études littéraires, Université du Québec à Montréal ; directeur fondateur de Figura, le Centre de recherches sur le texte et l'imaginaire, ainsi que du NT2, le Laboratoire de recherches sur les œuvres hypermédiatiques

RÉSUMÉ

L'industrie cinématographique nous a donné, ces dernières décennies, une quantité impressionnante de films à caractère apocalyptique. Ces films couvrent l'ensemble des situations associées à une fin du monde, depuis les premières annonces de la fin jusqu'au monde d'après la fin. Les films oscillent entre des Apocalypses traditionnelles et des apocalypses intimes, de ces fins vécues sur un mode restreint et qui condensent le destin de l'humanité entière. Des films tels que The Tree of Life de Terence Malick (2010) et Melancholia de Lars von Trier (2011), qui seront décrits ici, sont de telles apocalypses intimes qui se tiennent en retrait des spectacles à grand déploiement. Malgré tout, d'importances différences les séparent, surtout en ce qui a trait à leur traitement de la fin et de ses significations. Car, si le premier s'ouvre sans compromis à un imaginaire chrétien, où la fin est envisagée dans la perspective d'une renaissance à Dieu, suggérant de façon à peine voilée que la vie se poursuit après la mort, le second engage une posture résolument athée et déclare plutôt, dans un matérialisme radical, que toute vie cesse une fois la mort venue. Ces films engagent des imaginaires de la fin distincts, I'un, un imaginaire traditionnel à bien des égards et teinté de transcendance, l'autre, un imaginaire contemporain, de facture nihiliste.

MOTS-CLÉS: apocalypses - cinéma - imaginaires 
The film industry has given us, these last twenty years an impressive amount of apocalyptic movies. These films cover all situations associated with the end of the world, from the first prophetic announcement of the end to post-apocalyptic worlds. The stories told range from traditional Apocalypses, where worlds are destroyed, to intimate apocalypses deployed in a limited and subjective mode, where the fate of a few human beings or of one individual condenses the destiny of mankind. Films such as Terence Malick's The Tree of Life (2010) and Lars von Trier's Melancholia (2011) are such intimate apocalypses. Although both films shy away from mainstream disaster movies, fundamental differences separate them. My aim here is to show some of these differences, which have to do with the way the films represent the end and define its limits. For, if The Tree of Life engages in a Christian imagination, where the end is seen in the context of a spiritual rebirth, suggesting that life continues after death, Melancholia commits to a resolutely atheist posture and claims rather, in a radical materialism, that all life ceases after death. These films engage in distinct apocalyptic imaginations, the first, a traditional imaginary, suffused with transcendence, the second, a contemporary imaginary, nihilistic in its stance.

KEYWORDS: apocalypses - cinema - imaginary

Le cinéma nous a donné, ces dernières décennies, une quantité impressionnante de films à caractère apocalyptique. La fin du monde fait vendre. L'industrie cinématographique adore cette recette et multiplie à l'envi les blockbusters apocalyptiques. Un site comme Apocalypticmovies.com recense, par décennie, les films, toutes catégories confondues, qui traitent de la fin du monde. Pour la première décennie du vingtième siècle, 72 films et séries télé ont ainsi été identifiés (consulté le 27 décembre 2013); 29 autres ont été répertoriés pour la deuxième décennie. Le répertoire commence avec les années 1950, où 7 films sont identifiés, dont l'étonnant The Day the Earth Stood Still $(1951)^{1}$. Si on pensait que la fin du monde avait été désamorcée avec le passage au vingt-et-unième siècle, qui s'est produit sans effusion de sang ni bogue majeur, le début de ce siècle marqué par les attentats du 11 septembre et les multiples conflits qu'ils ont engendrés connaît une réactualisation de cet imaginaire de la fin².

Sur le site Apocalypticmovies.com, les œuvres sont classées en fonction de leurs sous-genres. Dans une des sections, on trouve même un sondage qui nous demande quel est notre genre apocalyptique favori. Nous avons le choix entre le post-apocalyptique, les zombies, les films de météores ou de comètes, la menace nucléaire, les pestes et les désastres naturels. Ces catégories témoignent d'un classement rudimentaire, soumis à une hollywoodisation, c'est-à-dire à une commercialisation excessive, tournée vers le spectaculaire, 
des causes et des conséquences de la fin du monde. Et à ce jeu, ce sont les films post apocalyptiques $(47,6 \%)$ et les films de zombies $(24,3 \%)$ qui ont la cote, raflant presque les trois quarts des réponses.

L'empan couvert par la cinématographie contemporaine participant de cet imaginaire de la fin est large. En termes narratifs, d'une part, les films couvrent l'ensemble des situations associées à une fin du monde, depuis les premières annonces de la fin jusqu'au monde d'après la fin. À une extrémité, un film tel que Take Shelter (Nichols, 2011) nous montre un prophète en devenir, dont les actions sont interprétées d'abord et avant tout comme les signes précurseurs non pas tant d'une fin du monde que d'une schizophrénie, héritée possiblement de la mère. À l'autre extrémité, dans The Road (Hillcoat, 2009), film adapté du roman éponyme de Cormac McCarthy de 2006, I'on suit les derniers êtres vivants pour qui les autoroutes et les diverses voies qui traversent le pays apparaissent comme les derniers vestiges de la civilisation et l'ultime moyen de survie. Il est d'ailleurs intéressant de voir la signification associée à la route dans ce film, qui ne signifie plus les grands espaces et la liberté, ce qu'elle fait dans l'imaginaire américain et les Road Movies ${ }^{3}$, mais la société, le dernier rempart contre la barbarie et la mort.

Le spectre est aussi très large, d'autre part, en termes de spectacle, voire de mythes. Les films oscillent entre des Apocalypses traditionnelles, déployées à grandeur de monde, et des apocalypses intimes, pour reprendre l'expression de l'écrivain américain John Hawkes, de ces fins vécues sur un mode restreint, par conséquent, et qui condensent le destin de l'humanité entière ${ }^{4}$. Pour les premières, pensons à ce navet que fut 2012, de Roland Emmerich (2009), reposant sur la fin du monde annoncée dans le calendrier maya et qui se présente comme un banal film de catastrophes, avec arche de Noé en prime. Pour les secondes, Biutiful d'Alejando Gonzales Iñàrritu (2010) offre un bel exemple de réflexion sur la mort et le destin de l'humanité.

Des films tels que Biutiful ou encore The Tree of Life de Terence Malick (2010) et Melancholia de Lars von Trier (2011) sont des apocalypses intimes qui se tiennent en retrait des spectacles à grand déploiement où l'apocalypse est posée comme un spectre diabolique et parfois grandguignolesque. La fin circonscrite mais beaucoup plus concrète du sujet, la sienne propre ou celle de son monde, y prend le relais pour exprimer de façon beaucoup plus précise l'abîme qui s'ouvre quand sont aperçues les limites de la vie. Le collectif et le singulier s'y articulent comme les faces opposées d'une même réalité imaginaire. Et ce que ces fictions perdent en spectacle, sur le plan de la représentation, elles le regagnent en efficacité symbolique. Leur propos n'est plus littéral, il devient allégorique. The Tree of Life tout autant que Melancholia ouvrent leur propos au devenir humain. Les deux films reposent sur un système d'échos entre histoire individuelle et ce destin plus large de la création et de la destruction du monde. Ce qui se joue dans la famille O'Brien, ainsi que dans la famille de Justine et de Claire, est abordé de façon minutieuse, sans compromis. Ce sont des drames familiaux qui secouent les êtres et les font vaciller, deuil ou mariage raté, mais ces drames sont enchâssés dans une 
histoire plus large qui leur donne une signification. Le regard se fait d'autant plus chirurgical que les conséquences des situations sont extrapolées à la grandeur de l'univers. Le premier, The Tree of Life, nous apprend que, quelle que soit notre intelligence, nous sommes aveugles à notre propre réalité. Le second, Melancholia, montre très précisément que les suites de l'échec des institutions humaines, telles que le mariage, sont une fin de ce monde qu'elles expriment. Si le premier s'ouvre sans compromis à un imaginaire chrétien, où la fin est envisagée dans la perspective d'une renaissance à Dieu, suggérant de façon à peine voilée que la vie se poursuit après la mort, le second engage une posture résolument athée et déclare plutôt, dans un matérialisme radical, que toute vie cesse une fois la mort venue. Il n'y a rien après. Ce qui est étonnant, c'est de retrouver, presque la même année, deux films complexes, deux imaginaires de la fin aux axiologies à ce point divergentes. En fait, ces deux films engagent des imaginaires de la fin distincts, I'un traditionnel à bien des égards et teinté de transcendance, l'autre contemporain, de facture nihiliste, et c'est à montrer la différence entre les deux que je vais me consacrer maintenant.

\section{NOUS SOMMES AVEUGLES À NOTRE PROPRE RÉALITÉ}

Que viennent faire ensemble les dinosaures, une famille américaine des années cinquante, et un bord de mer à marée montante? Ils font se croiser ontogénèse et phylogénèse, de même que le séculier et le religieux. Le récit du deuil de la famille O'Brien, dans The Tree of Life, apparaît comme la pièce centrale d'un triptyque. Ce tableau, c'est le temps présent, l'actualité du vivant, avec cette myopie qui le caractérise. Que savons-nous de ce que nous vivons? Que comprenons-nous de ce que notre vie nous offre comme expérience? Ce tableau est occupé par des êtres qui vivent, se reproduisent, grandissent et meurent. Ce présent est un moment de confusion. Nous ne comprenons pas nécessairement ce que nous sommes, ce qui nous pousse à agir, quelle est cette violence qui nous habite, quelles sont nos pulsions. C'est un monde opaque, ce que la cinématographie de Malick rend bien, qui coupe court à toute explication, qui reste près des êtres pour épier leurs réactions, leurs incompréhensions, leurs violences. Nous sommes confrontés à un monde de sensations et de perceptions, un monde où nos mécanismes de compréhension sont détraqués. Nous sommes, nous spectateurs, face à The Tree of Life, comme les transcendantalistes américains étaient face à la nature, à la fois éblouis et médusés. Éblouis par la complexité du monde; médusés par la force de ses manifestations, et le dessein qu'on y aperçoit se profiler. Celui d'une intentionnalité à l'œuvre qui dépasse nos propres déterminations et nos capacités d'imagination. Le film nous le montre clairement, nous sommes aveugles à notre propre réalité, parce que celle-ci ne dépend pas de nous, mais d'une intention supérieure, dont la réelle présence nous est rendue manifeste à certains moments cruciaux. Le monde des O'Brien est un monde simple aux valeurs conservatrices bien ancrées dans cette Amérique 
prospère des années cinquante. Or, le père, joué par Brad Pitt, sera soumis à des épreuves semblables à celles de Job. Il perdra son fils, comme celui-ci avait perdu ses fils et ses filles. Les mêmes interrogations viendront déstabiliser Jack, le fils aîné, qui se remémorera son passé, de nombreuses années plus tard.

Les interrogations sont ouvertement religieuses et elles s'insèrent dans un tableau en trois parties qui en confirment la dimension eschatologique. Les deux autres tableaux du triptyque, ceux peints sur les volets extérieurs de l'œuvre et dont la fonction est de se replier sur le panneau central afin d'en assurer le sens, en identifiant son origine ainsi que son horizon ultime, ouvrent le présent et cette anamnèse qu'il permet, à ces deux autres temps qui en déterminent la forme générale, le passé et le futur. Temps qui doivent être compris non pas tant comme ces moments que des êtres humains peuvent connaître, mais comme ces temps participant du Grand Temps, qui définit la vie sur terre. À la gauche du tableau principal, on trouve ainsi le Grand Passé, à savoir l'origine du monde, avec la création des planètes, l'origine de la vie sur terre, les cellules, les dinosaures, la genèse de l'humanité. Ce que nous nommons d'ailleurs «humanité», et qui repose sur notre capacité d'avoir des rapports emphatiques avec les autres, d'être dans des relations d'aide, l'interprétation malickienne des origines nous apprend qu'elle est présente dès la période des dinosaures. L'humanité est préfigurée dans les actions des reptiliens, comme l'Ancien Testament préfigure dans ses divers chapitres les actions du Christ dans le Nouveau. La volonté de lier I'histoire humaine aux destins du monde, de tisser des liens entre onto- et phylogénèse, était présente dans un film tel que 2001: A Space Odyssey de Stanley Kubrick (1968), dont l'amplitude, tout comme celle de The Tree of Life, allait des débuts de monde à sa fin. Mais le propos de Malick est ouvertement chrétien, plutôt que scientifique comme celui de Kubrick. Nous n'allons pas vers une technologie surdéveloppée et, à la limite, dangereuse parce que devenue autonome, mais vers une réunion des éléments, vers une union sous le soleil de Dieu. Comme le signale David Sterritt, «From the prayers at the beginning to the sermon in the middle and the vision of heaven at the end, Malick's film is wrapped in a religiosity that secular humanists will find nostalgic and naïve. » (Sterritt, 2011, p. 52)

Le troisième tableau de The Tree of Life se tourne ainsi vers le Grand Futur, mais un futur interprété selon des prémisses chrétiennes. La fin, c'est le monde de la fin du monde, selon la version chrétienne, un monde où nous sommes avec et en Dieu, un monde intercalaire où les morts, ceux qui hantent notre passé, reviennent cohabiter avec les vivants, et tous nous nous rejoignons dans la lumière naturelle et omniprésente de Dieu. Ce monde est représenté par cet espace où tous les éléments sont enfin réunis, car à la fois l'eau, la terre, l'air et le feu y sont présents. Les figures qui y évoluent marchent sur une surface ensablée recouverte de quelques centimètres d'eau. Et elles baignent dans la lumière, une lumière grandissante, car les plans de cette séquence commencent au crépuscule et se continuent jusqu'à 
ce que le soleil brille de tous ses feux, avant de se résorber. Les multiples contre-plongées de cette séquence assurent l'élévation des figures qui évoluent bien souvent les pieds dans l'eau, les vêtements flottant au vent. Elles les grandissent et assurent leur association avec le ciel. Et la musique, dramatique à souhait - c'est la Grande Messe des morts de Berlioz (requiem opus 5) - , accentue le caractère numineux de l'expérience. Ce ne sont pas que des réminiscences de Jack, c'est une interprétation cinématographique du royaume de Dieu, qui s'actualise à la fin du monde, quand tout aura été dit et fait.

The Tree of Life met en jeu un imaginaire de la fin chrétien, inspiré du transcendantalisme américain, qui met en équilibre les tensions entre la vie humaine, dans ses imperfections et ses violences, et le devenir humain, éclairé par la présence du sacré.

\section{LA MÉLANCOLIE DÉTRUIT LE MONDE}

Tout autre est la fin du monde représentée dans Melancholia de Lars von Trier. La fin, la destruction de la planète, n'est pas jouée sur un mode chrétien et sacré, mais nihiliste et à forte teneur psychanalytique. La planète qui menace la terre et qui finit par s'abattre sur elle se nomme Mélancolie. Cet état dépressif s'incarne en une planète, dont la trajectoire, au lieu de contourner la terre et de repartir compléter sa course dans l'espace sidéral, I'amène à frapper de plein fouet, après une double boucle, la terre. C'est une danse de la mort, nous dit le film. Une danse des planètes, sombre et agitée, qui se clôture par une annihilation complète du monde. «La mélancolie se caractérise», nous explique Freud, «par une dépression profondément douloureuse, une suspension de l'intérêt pour le monde extérieur, la perte de la capacité d'aimer, l'inhibition de toute activité et la diminution du sentiment d'estime de soi qui se manifeste par des autoreproches et des auto-injures et va jusqu'à I'attente délirante du châtiment. » (Freud, 2004, p. 8)

Ce diagnostic décrit bien le comportement de Justine, quand s'ouvre la deuxième partie du film. Après l'échec de son mariage et de la fête qui devait en couronner le déroulement, Justine se défait littéralement. Elle ne parvient même plus à sortir de chez elle. «She's ill, » déclare Claire à son mari ulcéré. Quand elle arrive enfin chez Claire, c'est à peine si elle parvient à marcher. Elle s'effondre sur le lit, doit être littéralement soulevée pour prendre son bain et ne parvient plus à manger. Ses propos sont incohérents et elle pleure à la moindre occasion. Le portrait serait caricatural si la cinématographie n'était aussi efficace, avec son esthétique d'ombre et d'obscurité, et si les enjeux ultimes du film, la fin du monde, ne venaient alourdir la moindre situation. Car la mélancolie n'est pas qu'une blessure ouverte pour Justine, elle prend la forme d'une planète. Mélancolie devient la figure même de la dépression qui menace Justine. Qui menace tout son monde. La métaphore s'est littéralisée. Le soleil noir de la dépression est devenu un corps céleste qui a la terre entière dans sa mire. 
La planète Mélancolie se cachait derrière le soleil, dit Léo, le fils de Claire, à Justine, prostrée sur son lit. Et maintenant elle nous effleure. La crainte, c'est bien qu'elle n'effleure pas la terre, mais la percute. Et cette appréhension, que Justine somatise, Claire la fait sienne. La mélancolie qui la tenaille est une véritable boule. John, son mari, tente de la rassurer, en lui disant que le passage de la planète sera la plus belle expérience de leur vie, mais ses paroles ne convainquent guère. Mélancolie n'a touché ni Mercure, ni Vénus, tel que promis, elle ne touchera pas la terre, dit-il. Mais ses arguments, fondés sur des analyses scientifiques, trouvent peu d'échos auprès de Claire, qui $s^{\prime}$ informe sur des sites Internet. Discours scientifique et discours religieux, à caractère prophétique, sont ainsi mis en tension. Et le suicide de John, quand le choc s'avèrera inéluctable et les calculs mathématiques se montreront erronés, sera la preuve que la crise dépasse les possibilités humaines de compréhension et de prévision.

Si la fin du monde suit l'échec du social et de son ordre, dans l'enchaînement chronologique des événements tout autant que dans le jeu des conséquences philosophiques, elle entraîne aussi un désaveu de la science et de sa représentation du monde. Les calculs sont faux, Mélancolie s'abattra sur terre: John ne peut accepter ce fait, d'où son retrait de la scène. Il ne reste alors, pour assister à la catastrophe, que la sœur, la mère et le fils qui se réuniront dans un rituel, l'ouverture d'un espace imaginaire, sorte de cercle magique ${ }^{5}$, qui leur servira d'interface, afin de comprendre, de s'approprier minimalement l'inacceptable, la fin de soi et de son monde.

Il est fascinant d'observer, dans cette deuxième partie, l'inversion des rôles. Si Claire sait vivre - c'est elle qui prépare le mariage, qui veille sur son fils et sa sœur-, elle ne sait pas mourir. C'est Justine qui le sait. La mort est son domaine. Le vide, son univers de prédilection. Quand Mélancolie s'approche dangereusement, c'est Justine qui prend en charge la famille et qui trouve les rituels nécessaires pour atténuer la violence du choc final. Elle sait. Et son savoir, fondé peut-être sur une connaissance intime de la mélancolie et du deuil, lui permet de rester lucide jusqu'à la fin. Elle sait ainsi que nous sommes seuls dans l'univers, seuls et sans dieu, seuls à exister.

(Justine) The earth is evil. We don't need to grieve for it.

(Claire) What?

(Justine) Nobody will miss it.

(Claire) But where would Leo grow up?

(Justine) All I know is, life on earth is evil.

(Claire) There may be life somewhere else.

(Justine) But there isn't.

(Claire) How do you know?

(Justine) Because I know things. (...) I know we're alone.

(Claire) I don't think you know that at all.

(Justine) 678. The bean lotery. Nobody guessed the amount of beans in the bottle. 
(Claire) No that's right.

(Justine) But I know. 678.

(Claire) Well perhaps. But what does that prove?

(Justine) That I know things. And when I say we're alone, we're alone.

Life is only on earth. And not for long.

Nous sommes seuls. Il n'y a pas de survie possible. La fin est une interruption. Elle n'a aucun sens, elle vient tout annuler, et de façon définitive. Il n'y a pas de parousie, pas de paradis, pas de justes, pas de réunion entre ciel et terre. Tout au plus y a-t-il une ouverture vers I'en-soi, vers cette grotte magique qu'invente Justine pour calmer Leo. Cette caverne, I'ultime repli, c'est l'imaginaire, le début du sacré. D'ailleurs le savoir de Justine s'apparente à celui d'un prophète. Elle sait, comme les prophètes savent. Son assertion nihiliste, et radicalement matérialiste, est teintée par ce savoir d'origine incertaine qui dépasse les contraintes humaines habituelles. Que sait-elle? Qui est-elle exactement? À moins qu'elle ne soit elle-même mélancolie, comme une muse, une humeur faite chair, au même titre que la planète.

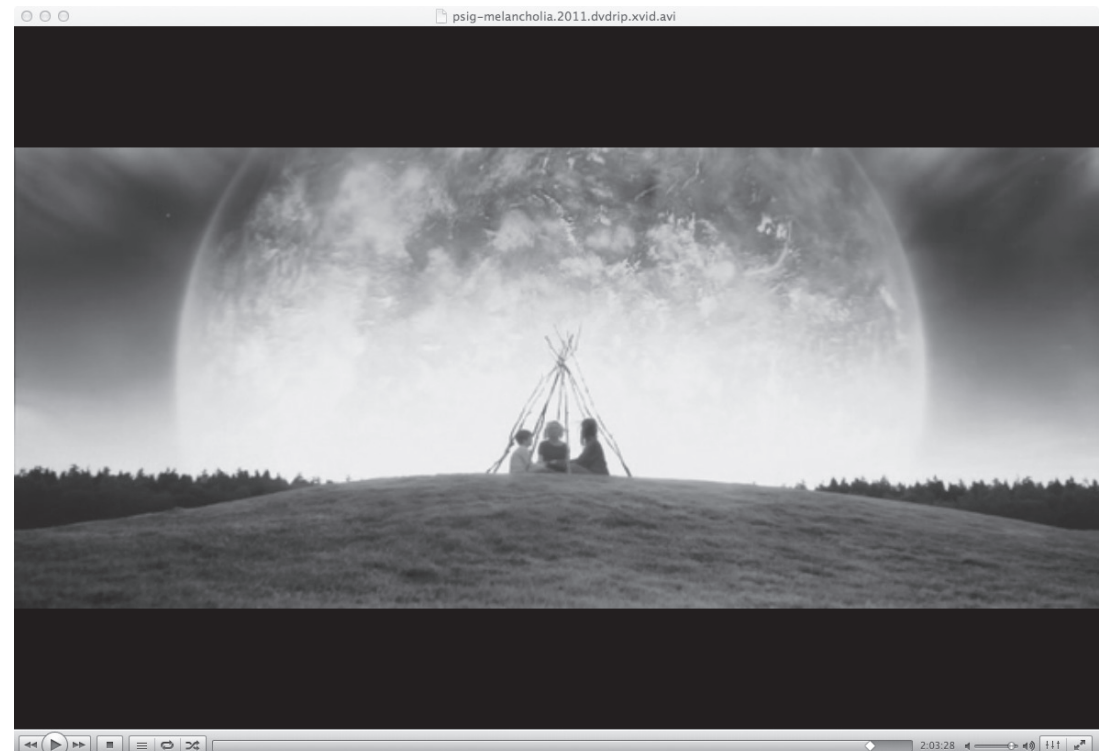

Justine n'est pas mélancolique, elle est mélancolie, ce qui explique son savoir sur tout ce que fait la planète. La figure s'est multipliée, elle s'est généralisée. Et la dimension allégorique est engagée. Mélancolie, c'est la planète, Justine et la maladie. Et rien ne survit à son passage. Le monde se clôt, dans le choc des corps, ce réel dont on tente de se parer en se construisant une demeure symbolique, une grotte magique faite de branches d'arbre, dont l'efficacité est suprêmement nulle face aux forces déchaînées par la collision. 


\section{INTERRUPTION ET \\ RÉPÉTITION : UN IMAGINAIRE \\ DE LA FIN CONTEMPORAIN}

Avec Melancholia, nous sommes dans une logique de l'interruption. La fin n'est pas la suite logique d'un monde en pleine décadence ou alors engagé dans une course à l'autodestruction. Elle ne se justifie pas en termes religieux, ce n'est pas une Apocalypse, ni même en termes scientifiques, la trajectoire de la planète défie les calculs, elle survient comme pur accident, elle s'impose comme interruption. Ce faisant, l'imaginaire de la fin qui y est à I'œuvre participe d'une logique autre, contemporaine, si tant est que, par ce terme, on renvoie à un régime d'historicité singulier ${ }^{6}$. C'est à un imaginaire de la fin contemporain auquel nous avons droit, imaginaire qui fait de l'interruption et de la répétition ses principes dominants.

Les manifestations narratives, fictionnelles et symboliques de l'imaginaire de la fin expriment, de façon explicite et parfois même exagérée, les effets dévastateurs des situations de crise, qu'elles soient individuelles ou sociales. Ces situations se vivent toujours au présent, qui apparaît, de ce fait, singulier. Les crises sont toujours perçues et vécues comme exceptionnelles, uniques et spécifiques. Elles imposent leur vérité, qui apparaît d'emblée comme un indépassable horizon. Cette singularité ressentie favorise paradoxalement leur reprise, chaque nouvelle crise effaçant le souvenir de la précédente. Or, si l'imaginaire de la fin contemporain se détache de ses prédécesseurs, traditionnels et modernes, c'est bien dans la multiplication et la réitération des situations de crise, provoquant une mutation fondamentale de son dynamisme même. Et c'est notre conception du temps qui en ressort perturbée.

La crise est une forme dynamique : elle tend vers sa résolution. Elle se déploie en effet en fonction d'une fin qui est sa loi. Le présent de la crise, s'il est oppressant et fascinant tout à la fois, dans ses effets de surface et l'exacerbation des perceptions qu'il entraîne, vient forcément à passer. Il se déverse dans le passé, qui en efface peu à peu les excès et les scories, et s'alimente d'un futur qui le tire résolument vers un ailleurs régénérant. Dans l'imaginaire contemporain, ce présent de la crise se transforme en réservoir qui ne laisse plus rien passer et qui s'emploie plutôt à tout ressasser. La crise est réitérée comme motif majeur.

Le site apocalypsemovies.com, par l'incroyable masse de films répertoriés, près de 80 depuis le passage au vingt-et-unième siècle, nous montre, par l'absurde, que la crise ne cesse d'être représentée et que la fin du monde qu'elle engage est une véritable obsession. Si, pris individuellement, chacun de ces films puise à des formes traditionnelles de récits de fin du monde, regroupés ils décrivent un autre état de l'imaginaire, où la répétition s'impose comme trait dominant, et l'interruption, comme seule façon de mettre fin au cycle. La fin y apparaît comme une frontière qui ne peut être traversée et qui est gardée en vue. C'est dire que la crise ne se résout jamais, sa réalité ne 
cesse d'être réitérée. Et cette répétition, le cercle vicieux d'une fin appréhendée et, soit repoussée, soit rejouée, substitue l'épuisement à la transcendance. Ce qu'on voit alors s'imposer, c'est une transformation des traits de I'imaginaire de la fin. Dans ce passage, c'est la façon même dont on conçoit notre monde et notre temps qui se modifie, à la manière d'un changement de paradigme. Et la nouvelle conception qui s'implante, on peut la décrire comme un régime contemporain d'historicité. On a vu apparaître, tout au long du siècle dernier, un tel régime qui s'oppose à un régime moderne. Pour Hartog, un régime d'historicité est «un cadre de pensée de longue durée, une respiration, une rythmique, un ordre du temps, qui permet et qui interdit de penser certaines choses. » (Hartog, 1995, p. 1222) Il est un filtre par lequel nous effectuons nos projections et interprétations, c'est-à-dire un interprétant à portée générale et d'une grande efficacité qui conditionne le déroulement de nos processus imaginaires. On ne comprend le monde, on ne le représente qu'en fonction d'une conception préalable qui en sert de canevas. Or, le régime qui nous est propre surdétermine le rôle du présent, mais d'un présent essentiellement anxiogène, dont le mode fondamental de connaissance serait mélancolique.

L'imaginaire de la fin est un révélateur intéressant de ce passage à un régime contemporain d'historicité, car ce changement de paradigme en transforme de façon importante les manifestations. La principale modification est le fait qu'en régime contemporain, la fin n'apparaît plus comme un principe de cohérence, mais comme une manifestation du chaos ou du désordre. La fin $n^{\prime} y$ est plus une conclusion, mais une interruption; elle n'ouvre plus à une transcendance, mais à une répétition stérile. De linéaire, l'imaginaire de la fin devient interruptif, et l'arc entier qui nous fait tendre du passé vers l'avenir se disloque, le présent occupant l'espace entier de la conscience. La fin devient une impasse, et la transition qui fait passer à un monde nouveau et rénové s'enraye. De l'opposition traditionnelle entre fin cyclique et fin linéaire, qui permettait de distinguer un imaginaire traditionnel, fondé sur le religieux, et un imaginaire de la fin moderne, essentiellement profane, nous sommes passés à une opposition entre la fin comme principe de cohérence et la fin comme simple interruption, jeu des contingences.

L'interruption, et sa conséquence, la répétition, sont représentatives de l'imaginaire contemporain. La fin ne s'y ouvre plus sur aucune transcendance permettant de rétablir l'ordre. Au contraire, cette fin se retourne sur ellemême, créant un pli dont on ne peut s'extraire ou alors dont on est toujours déjà exclus. Si l'imaginaire de la fin moderne tablait sur une mémoire capable d'embrasser tous les temps et de maîtriser leurs relations, l'imaginaire de la fin contemporain mise plutôt sur l'oubli comme modalité de l'agir, apte à survivre aux inconstances du temps et de ses perturbations. Et l'oubli est à l'origine d'une certaine forme de répétition: non pas celle qui engage à la perfection, et qui repose sur la mémoire, sur l'exactitude du geste et la recherche d'une transcendance, mais celle qui s'ouvre à une dégradation, à une reprise de moins en moins fidèle, mais toujours aussi obsédante de la 
même situation. Les gestes ne sont pas repris comme moyen visant un but précis, mais comme habitude dont on ne parvient plus à se déprendre.

La tension entre The Tree of Life et Melancholia est celle entre un imaginaire de la fin moderne, replié sur un schéma traditionnel chrétien, qui fait de la fin le passage à un autre monde, et un imaginaire de la fin contemporain, où celle-ci est une interruption qui ne valide rien et ne s'inscrit dans aucun métarécit assurant la cohérence du monde. Les deux films se rejoignent par contre dans l'exploration d'un présent qui, pour leurs protagonistes, s'impose comme un pur moment de crise. L'effet de répétition apparaît non pas tant dans la confrontation de leurs choix narratifs que dans leur coprésence dans un même état social, et dans un corpus cinématographique qui fait du motif de la fin une donnée essentielle de notre époque, déclinée en récits post-apocalyptiques, films de zombies et de catastrophes, apocalypses intimes, attentats nucléaires, pestes, etc. Leur coprésence témoigne d'un imaginaire crépusculaire, où la répétition est la seule façon de contrer l'interruption et de redonner un semblant de cohérence à ce qui en semble manifestement dépourvu.

\section{BIBLIOGRAPHIE}

BENOLIEL, B. et J.-B. Thoret (2011). Road Movie, USA, Paris, Éditions Hoebëke, 2011.

CHASSAY, J.-F. (2008). Dérives de la fin: sciences, corps et villes, Montréal, Le Quartanier.

CHASSAY, J.-F., A. E. Cliche et B. Gervais, (dir.) (2005). Des fins et des temps. Les limites de l'imaginaire, Montréal, Figura, $\mathrm{n}^{\circ} 12$.

GERVAIS, B. (2009). L'imaginaire de la fin: temps, mots et signes. Logiques de l'imaginaire. Tome III, Montréal, Le Quartanier.

FREUD, S. (2004). «Deuil et mélancolie. Extrait de Métapsychologie», Sociétés, 2004, n 86, p. 7-19.

HARTOG, F. (2003). Régimes d'historicité: Présentisme et expérience du temps, Paris, Éditions du Seuil.

HARTOG, F. (1995). «Temps et Histoire», Annales HSS, n 6, p. 1219-1236.

HUIZINGA, J. (1988). Homo ludens, essai sur la fonction sociale du jeu, Paris, Gallimard.

STERRITT, D. (2011). «Days of Heaven and Waco: Terrence Malick>s The Tree of Life», Film Quarterly, 2011, vol. 65, n²1, p. 52-57.

\section{NOTES}

1 Le site ne se limite pas aux films déjà diffusés, il suit l'actualité cinématographique, les films et séries télé en production. Il donne aussi des informations sur des livres et des romans. Un forum est ouvert. Plus qu'une simple base de données, le site est un environnement de recherches et de connaissances.

2 Un projet tel que le Lower Manhattan Project, de l'Équipe de recherche sur I'imaginaire contemporain (ERIC LINT), offre un panorama des productions littéraires et cinématographiques qui traitent des attentats du 11 septembre 2001. La dimension apocalyptique y transparaît sans peine. Voir http:// Imp.uqam.ca/ 
3 Sur cette question, lire notamment Road Movie, USA, de Bernard Benoliel et Jean-Baptiste Thoret (Paris, Éditions Hoebëke, 2011).

4 J'ai exploité cette distinction dans L'imaginaire de la fin: temps, mots et signes. Logiques de I'imaginaire. Tome III, Montréal, Le Quartanier, 2009. On peut se référer aussi à I'essai de Jean-François Chassay, Dérives de la fin : sciences, corps et villes, Montréal, Le Quartanier, 2008).

5 C'est la notion initialement définie par Johan Huizinga dans Homo ludens, essai sur la fonction sociale du jeu, Paris, Gallimard, 1988.

6 François Hartog présente cette notion dans Régimes d'historicité: Présentisme et expérience du temps, Paris, Éditions du Seuil, 2003. 